10.2478/gb-2020-0019

sciendo

\title{
Zur Anwendbarkeit einiger Konzepte der Theaterpädagogik im Literaturunterricht an einigen Beispielen
}

\author{
Larisa PIOARU \\ Drd., Transilvania-Universität, Kronstadt/Braşov; \\ E-Mail: larisa.pioaru@unitbv.ro
}

\begin{abstract}
The paper presents the applicability within literature classes of some concepts borrowed from theater pedagogy. It highlights a few teaching and learning strategies, which facilitate a better text comprehension and most of all inspire fun by learning and by working with texts, and it is based on precise examples, respectively projects of students from Transilvania University in Brasov.
\end{abstract}

Keywords: theater pedagogy, literature classes, text comprehension.

\section{Theaterpädagogik - kurze Darstellung des Konzeptes}

Die Gesellschaft befindet sich in einem ständigen Entwicklungsprozess und wir müssen uns zweifellos daran anpassen. Die Erziehung (in der Familie) und die Ausbildung (in der Schule) spielen eine sehr wichtige Rolle in diesem Prozess. Diese zwei Elemente sind unentbehrlich in der Gestaltung und in dem Fortbestehen jeder Gesellschaft. Wiederum ist die Gesellschaft dafür verantwortlich, eine harmonische Entwicklung des Individuums, einerseits in der Familie, andererseits in der Schule, zu ermöglichen. Ebenso muss das Ausbildungssystem regelmäßig aktualisiert werden, so dass es den Bedürfnissen der neuen Generationen von Schüler entspricht. 
Da die Schüler ziemlich schwer anzuregen sind, muss man ständig innovative, vergnügliche und unterhaltsame Wege finden, um bei ihnen den Spaß am Lernen zu wecken. In dem Literaturunterricht zum Beispiel kann der Lehrer verschiedene Methoden aus der Theaterpädagogik übernehmen und verwenden. Das Gleiche gilt auch für andere Schulfächer, aber der vorliegende Beitrag bezieht sich auf die Verbindung zwischen Theaterpädagogik und Literaturunterricht.

So wie Mirona Stănescu in ihrer Dissertation ${ }^{1}$ erwähnt, spielt das Theater schon seit dem 17. Jahrhundert eine wichtige Rolle in der Schule, indem es „moralisch-didaktische Ziele"2 setzt. Beginnend mit der Reformpädagogik von Anfang der 1920er Jahre spricht man von Laienspiel und Schulspiel, die eine „ästhetische und künstlerisch-handwerkliche"3 Dimension bekommen. Die 1950er Jahre waren die Phase der musischen Erziehung und in den 1960er Jahren erscheint das darstellende Spiel, welches sich ,von der Tradition der Musischen Bildung"4 entfernt. „Das Spiel soll den Schülern die Möglichkeit geben, sich mithilfe der verschiedenen Darstellungsmöglichkeiten mit der Realität auseinanderzusetzen und soziale Haltungen auszuprobieren."

Die Theaterpädagogik als selbstständige Disziplin entsteht erst nach 1980 und sie ,entwickelt sich [...] weg von einem Theater, dass die Menschen im Theaterspiel über das ,richtige' Leben aufklären wollte, hin zu einem Theater, indem die individuelle Erfahrung zählt, bis zu der Entdeckung der ästhetischen Erfahrungsmöglichkeiten im Theaterspiel." ${ }^{\circ 6}$

1 Stănescu, Mirona: Theaterpädagogik in Rumänien. Hamburg: Dr. Kovač Verlag. 2012, S. 13.

2 Ebd., S. 16.

3 Ebd.

${ }^{4}$ Ebd.

5 Ebd., S.13.

${ }^{6}$ Hagl, Elisabeth: Theaterpädagogik im Kontext einer lebendigen Lernkultur. Magisterarbeit im Rahmen des Studiengangs Pädagogik an der 
Die Theaterpädagogik richtet ihr Augenmerk auf die ästhetische Dimension des darstellenden Spieles. ${ }^{7}$ „Theaterpädagogik mit Fokussierung auf die Ästhetik umfasst das Zuschauen und das praktische Erlernen theaterpädagogischer Arbeitsweisen und Formen. Schüler lernen mit verschiedenen theatralen Aus-

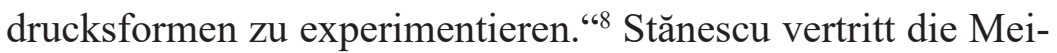
nung, dass ,das wesentliche Ziel der Theaterarbeit mit Schülern die persönliche, ästhetische und soziale Bildung der Schüler"9 sei.

In der Veröffentlichung des Bund(es) Deutscher Amateurtheater: Resolution ,Kinder spielen Theater ${ }^{10}$ " wird einerseits die Idee der ästhetischen und kulturellen Bildung durch das Theater mit all den Aspekten von Theaterarbeit vorangetrieben und andererseits weist man auf die Einbeziehung der Schüler als aktive Teilnehmer an dem Bildungsprozess hin:

Theater mit Kindern muss zum unverzichtbaren Bestandteil ästhetischer Bildung werden - überall in Deutschland. Das Kind erlebt sich im Theaterspiel als handelndes Subjekt, das sich zu Menschen und zur Welt als Ich in Beziehung setzt. Dem Feld der ästhetischen Frühförderung kommt eine prägende Rolle zu, denn ästhetische Erfahrungen und die sinnlich-kreative Praxis sind Ausgangspunkt aller Selbst- und Welterfahrung. Die lebenslange Neugier auf Kunst und Kultur muss in der Kindheit geweckt und mit immer neuen Impulsen lebendig gehalten werden. Kulturelle Bildung als

Ludwig Maximilian-Universität München, vorgelegt am 4.10.2006, S. 13, https://www.yumpu.com/de/document/read/3541843/theaterpadagogik-im-kontext-einer-lebendigen-lernkultur (Zugriff am 21.06.2019).

7 Vgl. Stănescu 2012, S. 13.

8 Ebenda, S. 14.

9 Ebenda.

${ }^{10}$ Hoffmann, Klaus/ Radermacher, Norbert/ Reiss, Joachim u.a.: Bund Deutscher Amateurtheater: Resolution „Kinder spielen Theater“. In: Deutscher Kulturrat (Hrsg.): Kulturelle Bildung in der Bildungsreformdiskussion. 2005, S. 418-419. https:/www.kulturrat.de/wp-content/ uploads/2016/04/konzeption-kb3.pdf (Zugriff am 27.06.2019). 
lebenslange Bildung setzt voraus, dass sie früh beginnt und damit als integraler Bestandteil von Bildung und Leben erfahren wird. Dass Kinder Theater spielen, ist ein wichtiger Bestandteil der ästhetischen Bildung. „Kinder spielen Theater" heißt auch, dass Kinder Theater sehen und dass die Produktion und Rezeption von Kunst im Bildungsprozess untrennbar miteinander verbunden sind. ${ }^{11}$

\section{Das darstellende Spiel}

„D[arstellendes] S[piel] ist eine Bezeichnung für das Schulfach Theater, welches die traditionellen Unterrichtsfächer des Lernbereichs $\rightarrow$ Ästhetische Bildung, Kunst und Musik ergänzt." 12 Carmen Puchianu definiert das darstellende Spiel folgendermaßen:

Das Spiel stellt das Erfassen, Gestalten und Umgestalten einer Handlung/Situation in eine andere dar, die sich zunächst durch einen gewissen Grad an Scheinhaftigkeit kennzeichnet, jedoch an einen eigens bestimmten Raum und eine ebensolche Zeit gebunden ist, beziehungsweise nach Regeln ordnungsgemäß verläuft und Gemeinschaftsverbände ins Leben ruft, letztendlich aus den Gewohnheiten, also dem Alltag hervortreten sind. ${ }^{13}$

Die Autorin betont, welche Rolle der Lehrkraft in diesem Verfahren - im Rahmen des Literaturunterrichtes - zukommt, und zwar die Rolle „des Moderators, sprich Koordinators und Beraters" 14 und sie stellt wichtige Hinweise zur Verfügung: Die Lernenden sollten kleine Arbeitsgruppen bilden und die Lehr-

${ }^{11}$ Ebenda, S. 418-419.

${ }^{12}$ Darstellendes Spiel. In: Wörterbuch der Theaterpädagogik. Hrsg. von Deutsches Archiv für Theaterpädagogik, DATP, Schibri Verlag 2003. Unter: http://www.archiv-datp.de/worterbuch-darstellendes-spiel/ (Zugriff am 27.06.2019).

${ }^{13}$ Puchianu, Carmen: Das Schulspiel im Deutschunterricht und ausserhalb desselben. Methodisch-didaktische Erwägungen. Ein Handbuch für Lehrer. Kronstadt: Aldus Verlag 1996, S. 6.

${ }^{14}$ Ebd., S. 11. 
kraft soll einerseits die Lernenden anregen sich auch individuell für das Spiel vorzubereiten, ${ }^{15}$ andererseits muss sie ihnen erklären, dass es wichtig ist

etwas in Zusammenarbeit zu erstellen und eine gemeinsame Verantwortung zu tragen. Recht bald werden sie merken, daß das Endergebnis sehr verschieden ausfallen kann, daß es nicht egal ist, ob alle oder nur einer oder zwei aktiv sind. Sie werden merken, daß in solchen Fällen der Beitrag jedes Einzelnen zählt, wenn die Gruppe ein gutes Ergebnis erreichen will. ${ }^{16}$

Wenn man das darstellende Spiel als didaktische Methode verwendet, lernt man mit allen Sinnen, d.h. nicht nur mit den Gedanken, sondern auch mit dem Körper. Laut Puchianu, ist es wichtig „den Text sozusagen mit dem ganzen Körper zu erfahren, zu erfassen und zu verstehen, indem man Text und Sprache in Körperempfinden umsetzt." ${ }^{\prime 17}$ Was die Arbeit mit dem Text betrifft, hebt Puchianu eine Besonderheit des Dramentextes im Vergleich mit anderen Gattungen hervor, nämlich seine performative Dimension:

Spricht man über Theater, bezieht man sich zuallererst auf das Theaterstück, d.h. auf das Drama, sei es in geschichtlichem, sei es in gattungsspezifischem Kontext. Im Mittelpunkt der Beschäftigungen steht dementsprechend der Dramentext als Form der Dichtung, seine Bedeutung wird hinterfragt und man bemüht sich grundsätzlich um die Interpretation desselben, wie man sich um die Interpretation eines Gedichtes oder eines Romans bemühen würde. Dabei verliert man jedoch das Wesentliche aus den Augen und zwar, dass der Dramentext nicht ausschließlich seinetwegen, sondern seiner Aufführung wegen geschrieben worden ist. Ohne seine direkte Anbindung an

${ }^{15}$ Vgl. ebd.

${ }^{16}$ Puchianu, Carmen: Das Schulspiel im Deutschunterricht und außerhalb desselben. Methodisch-didaktische Erwägungen. Ein Handbuch für Lehrer. Kronstadt: Aldus Verlag 1996, S. 11.

${ }^{17}$ Puchianu, Carmen Elisabeth: Roter Strick und schwarze Folie. Postmoderne Theateradaptionen auf den Leib geschrieben. Brasov: Universitatea Transilvania 2016, S. 136. 
und ohne seine Vervollkommnung durch das Theater bleibt jeder Dramentext eine bedeutungs- und lebensarme Hülle. ${ }^{18}$

Laut Puchianu ist diese theaterpädagogische Methode besonders wichtig, denn sie ermöglicht ein besseres Textverständnis, hilft den Lernenden Hemmungen abzubauen, sich zu äußern und eigene Meinungen $\mathrm{zu}$ formulieren, durch sie entwickeln die Lernenden kognitive und kommunikative Kompetenzen, diese Methode fördert die Textproduktion und Textrezeption, und nicht zuletzt macht sie Spaß und trägt dazu bei, dass authentische theatrale Begabungen entdeckt werden können. ${ }^{19}$

$\mathrm{Zu}$ illustrieren sind diese Thesen mit folgendem Unterrichtsprojekt, das vor einigen Jahren an der Transilvania-Universität in Brașov/ Kronstadt durchgeführt wurde. Dort gibt es ein theaterpädagogisches Wahlpflichtfach im Curriculum, das gelegentlich von Studenten gewählt wird und das aufgrund theaterpädagogischer Übungen literaturdidaktische Anregungen vermittelt.

Darstellendes Spielim Kreatives Schreiben, Wahlpflichtfach, BA3, 2. Semester, 2010-2011. ${ }^{20}$ Das Prüfungsthema war das Erstellen eines kurzen dramatischen Textes mit anschließender szenischen Umsetzung. Folgende Arbeitsanleitungen wurden erteilt:

In Form von Gruppenarbeit sollten die Studenten zu dritt oder zu viert in je einer Gruppe arbeiten, dafür erhielten sie als Arbeitshilfe ein Blatt Papier mit verschiedenen Stichwörtern, woraus sie einige auswählen sollten, um eine Geschichte mit dem Thema „Waldspaziergang“ zu gestalten. Die Studenten

${ }^{18}$ Ebd., S. 6.

${ }^{19}$ Vgl. Puchianu, Carmen: Das darstellende Spiel und sein Einsatz im Germanistikunterricht am Beispiel eines Projektes mit Kronstädter Germanistikstudierenden. Temeswar: Mirton Verlag 2008, S. 143, unter: http://www.diacronia.ro/ro/indexing/details/A13124/pdf (Zugriff am 28.06.2019).

${ }^{20}$ Dieses Wahlpflichtfach wurde inzwischen in das 2. Studienjahr BA, 1. Sem. verlegt., Vgl. www.unitbv.ro (Zugriff am 20.06.2019). 
sollten in ihrem Kurzprojekt darauf achten, dass sowohl die individuelle als auch die gemeinsame Arbeit berücksichtigt wird. Jede Gruppe hat zuerst das Sujet festgelegt, dann haben sie den Handlungsablauf zusammen erarbeitet, danach die Figuren festgelegt, und anschließend hat jedes Mitglied der Gruppe eine Rolle zugeteilt bekommen. Jeder sollte seine Figur auf persönliche Weise erstellen, ohne das Thema aus den Augen zu verlieren.

\section{Zur Bewertung des Projekts}

Die Bewertung des Abschlussprojektes zum Thema „Waldspaziergang" war die Aufgabe der Dozentin einerseits und der Studierenden andererseits. Die Dozentin hat jeden der Mitwirkenden nach seiner mündlichen Stellungnahme zum Projekt und nach dem individuellen Beitrag in der Gruppe bewertet. Die Gruppen haben sich untereinander nach drei Kriterien beurteilt: Originalität, Gesamtkonzept und Zusammenwirken der Gruppenmitglieder. Jedes Bewertungskriterium war 20 Punkte wert.

\section{Jeux Dramatiques, Playback-Theater, Szenische Interpretation}

In ihrem Vorgehen betreffend die Entstehung eines theaterpädagogischen Ausbildungskonzepts für die Babeș-Bolyai-Universität wählt und analysiert Mirona Stănescu theaterpädagogische Arbeitsweisen von acht Spezialisten: Jeux Dramatiques (Heidi Frei),Playback-Theater(GittaMartens), SzenischeInterpretation (Ingo Scheller und Marcel Kunz), Schwerpunkt: Sprache (Hans Martin Ritter), Schwerpunkt: Improvisation (Keith Johnstone), Elementarer Tanz (Maja Lex) und Kontaktimprovisation (Steve Paxton). Die ersten drei Konzepte entsprechen dem Literaturund Deutschunterricht und ,basieren auf ein ästhetisches 
Rezeptionsverfahren der Schüler"21, die nächsten vier sind eher für die Schauspielschulen geeignet. Da unser Beitrag auf den Literaturunterricht fokussiert ist, beziehen wir uns weiterhin auf die Ansätze von Frei, Martens, Scheller und Kunz. Der Hauptzweck dieser vier Herangehensweisen ist ,die Vermittlung von Literatur und das Darstellen von mündlichem Erzählen."22

Durch die szenische Interpretation möchten Scheller und $\mathrm{Kunz}^{23}$ den Schülern ein besseres Textverstehen ermöglichen. Freis Konzept der Jeux Dramatiques hat als Ziel „den Spielenden einen spielerischen Umgang mit Texten”"24 anzubieten und nicht unbedingt ein besseres Verstehen eines Textes zu gewährleisten. „Durch das Interpretieren der literarischen Vorgaben erhalten die Schüler die Möglichkeit, die vorgestellten Situationen mit den eigenen Erlebnissen und Erfahrungen zu verbinden und darüber zu reflektieren." ${ }^{25}$

Martens Methode des Playback-Theaters basiert auf dem Erzählen eines Erlebnisses, das dann durch theatrale Ressourcen und Methoden inszeniert wird. Diese Herangehensweisen gehören zu dem literarischen Lernen und sie bezwecken keine Aufführung eines Textes. ${ }^{26}$

Jeux Dramatiques oder das Ausdrucksspiel verfolgt den Zweck ,die individuellen Ausdrucksmöglichkeiten zu fördern, indem die Teilnehmer spontan ihre Fantasie und ihre inneren Bilder zum Ausdruck bringen. ${ }^{\text {" }}{ }^{27}$ Die Lernenden können anhand dieser Methode sowohl mit Prosatexten als auch mit lyrischen Texten arbeiten. Die Methode erweist sich als effizient, denn ,da es sich um das Selbsterlebte handelt, wird den Kindern das Sprechen und das Ausdrücken der eigenen Ideen

${ }^{21}$ Stănescu 2012, S. 41.

${ }^{22}$ Ebenda.

${ }^{23}$ Vgl. ebd., S. 41.

${ }^{24}$ Ebenda.

${ }^{25}$ Ebenda.

${ }^{26} \mathrm{Vgl}$. ebd.

${ }^{27}$ Ebenda, S. 44. 
leichter fallen. So werden sie Sicherheit in Bezug auf ihre Sprache bekommen." 28

Das Playback-Theater ist laut Martens, ,eine pädagogische Arbeit mit spielerischen Mitteln, deren Ziel die Aneignung von Erleben durch Kommunikation mit anderen ist, wobei das Spiel nicht nur dem Erzähler, sondern allen Kindern die Möglichkeit gibt, das Erlebnis des Einzelnen als Eigenes nachzuvollziehen. “29 Diese Methode fördert die Erzählfähigkeit und die Hörfertigkeit der Lernenden, aber auch die Fähigkeit das Erzählte anzunehmen. ${ }^{30}$

Ingo Scheller beabsichtigt durch die szenische Interpretation „vergessene, ausgegrenzte oder noch nicht entdeckte Gefühle, Wahrnehmungs- und Denkmöglichkeiten (wieder) bewusst zu machen und als Teil des eigenen Selbst zu akzeptieren. ${ }^{\text {" }}{ }^{31}$

Um die Lernprozesse im Rahmen des szenischen Spieles zu verwirklichen, muss man die Spielsituation ernsthaft entwerfen und den zuvor festgesetzten Zielen folgen. ${ }^{32}$ Ganz wichtig für die szenische Interpretation sind ,die Aktivierung der emotionalen Prozesse" ${ }^{\prime 3}$ und ,die Stimulierung der kognitiven Prozesse." ${ }^{34}$

Laut Scheller können anhand der szenischen Interpretation

soziale Situationen und Dramen, die in Erlebnissen, Theorien, Geschichten, Bildern und Texten entworfen und interpretiert werden, szenisch rekonstruiert, mit eigenen Erlebnissen durchsetzt und unter Aktivierung eigener Wünsche, Übertragungen und Verhaltensmuster

${ }^{28}$ Ebenda, S. 45.

${ }^{29}$ Martens, Gitta: ,, Wisst ihr, was gestern passiert ist? ” Kinder erzählen und spielen Playback-Theater. 2004, S. 4, zitiert nach Stănescu, 2012, dort S. 45.

${ }^{30}$ Vgl. Stănescu, 2012, S. 49.

${ }^{31}$ Scheller, Ingo: Szenisches Spiel. Handbuch für die pädagogische Praxis. Berlin: Cornelsen Verlag 1998, S. 25, zit. nach Stănescu, 2012, dort S. 51.

${ }^{32}$ Vgl. Stănescu, 2012, S. 51.

${ }^{33}$ Ebenda, S. 52.

${ }^{34}$ Ebenda. 
neu gedeutet werden, so dass sie in ihrer Entstehung, ihrem Verlauf und in der spezifischen Interpretation erfahrbar werden. ${ }^{35}$

Auch Marcel Kunz fördert die szenische Interpretation im Rahmen des Literaturunterrichtes. Die Lektüre wecke die Fantasie und die eigenen Erinnerungen der Schüler, so Kunz. ${ }^{36}$ Die Auffassung von Kunz fokussiert auf die Arbeit mit dem literarischen Text, aber „Es handelt sich [...] um eine ,produktive, experimentelle und gestaltende Auseinandersetzung"',37

Mirona Stănescu fasst mit Bezug auf die szenische Interpretation zusammen:

Die für das szenische Spiel verwendeten schauspiel- und theaterpädagogischen Spielverfahren ermöglichen es, Lern- und Erkenntnisprozesse zu initiieren, die den Lernenden die Möglichkeit geben, anhand der angenommenen Rollen innere und äußere Haltungen zu erkennen und sich ihrer bewusst zu werden. Ausgangspunkt ist einerseits der literarische Text und dessen ,fremde' Welt und andererseits die persönlichen Erlebnisse und Erfahrungen der Schüler, die zugleich Bezugspunkt für den Literaturunterricht werden. ${ }^{38}$

Das Hauptziel dieser vier Konzepte, Darstellendes Spiel, Jeux Dramatiques, Playback-Theater und Szenische Interpretation, ist ,ein ganzheitliches und handlungsorientiertes Lernen, ein Lernen mit allen Sinnen, denn nur in der Zusammenarbeit aller Sinne kann das Kind neue Erfahrungen und Erkenntnisse gewinnen"39, so Stănescu.

Die praktische Illustration der Anwendbarkeit obiger Konzepte stellt folgendes Unterrichtsprojekt dar: Textdidaktik, Wahlpflichtfach, BA 3, 2. Semester, 2011-2012.. ${ }^{40}$

${ }^{35}$ Ebenda, S. 53.

${ }^{36} \mathrm{Vgl}$. Ebenda.

${ }^{37}$ Ebenda.

${ }^{38}$ Ebenda, S. 55.

${ }^{39}$ Ebenda, S. 68.

${ }^{40}$ Dieses Wahlpflichtfach wurde in das 3. Studienjahr BA, 1.Sem verlegt, vgl. www.unitbv.ro (Zugriff am 20.06.2019). 
Das Prüfungsthema war das Umschreiben und die moderne Interpretation einer literarischen Vorlage, in diesem Fall die Ballade Die Brück' am Tay von Theodor Fontane, mit anschlieBender Inszenierung.

\section{Arbeitsanleitungen:}

Gruppenarbeit: Die Studenten sollten den Text szenisch umsetzen, indem sie bewegte Sequenzen mit Standbildern verknüpfen. Dazu hatten sie die Aufgabe die Ballade in einer persönlichen Manier umzuschreiben und eine unvorhergesehene Pointe zu finden. Jedes Mitglied der Gruppe hat eine bestimmte Aufgabe übernommen und so haben alle zu dem Erfolg des Projektes beigetragen.

\section{Zur Bewertung:}

Das Bewertungsraster hat sich auf sieben Kriterien bezogen. Fünf davon galten für das Projekt als Ganzes, nämlich inhaltliches Verständnis der Vorlage, Umsetzung des Balladentextes in einen szenischen Text,Zusammenwirken der Gruppenmitglieder (Teamarbeit), Gesamtkonzept und Originalität, während die letzten zwei Kriterien sich auf die individuelle Leistung jedes Mitwirkenden bezogen haben: die mündliche Stellungnahme und der individuelle Beitrag in der Gruppe. Bei jedem Kriterium konnten maximal 10 Punkte vergeben werden und die Gruppen haben sich gegenseitig bewertet.

\section{Fazit}

Die in der vorliegenden Arbeit vorgestellten theaterpädagogischen Konzepte weichen von den klassischen Unterrichtsmethoden ab, vor allem in Bezug auf den Literaturunterricht. Während das von Mirona Stănescu und Carmen Puchianu erwähnte Lernen mit allen Sinnen für die Theaterpädagogik 
geeignet ist, stellt es eine ganz innovative und kreative Herangehensweise für den Literaturunterricht im NachwendeRumänien, auch auf Hochschulniveau, dar.

Unseres Erachtens ist es sehr interessant und produktiv zu beobachten und zu untersuchen, wie diese Lernstrategien in dem Prozess der Literaturvermittlung wirken, wie sie die Ausbildung der Lernenden beeinflussen und vor allem, ob sie Zukunftsaussichten haben.

\section{Literaturverzeichnis}

Martens, Gitta: ,Wisst ihr, was gestern passiert ist?” Kinder erzählen und spielen Playback-Theater. In: gruppe \& spiel. Zeitschrift für kreative Gruppenarbeit 5/ 2004, Seelze.

Puchianu, Carmen: Das Schulspiel im Deutschunterricht und ausserhalb desselben. Methodisch-didaktische Erwägungen. Ein Handbuch für Lehrer. Kronstadt: Aldus Verlag. 1996.

Puchianu, Carmen Elisabeth: Roter Strick und schwarze Folie. Postmoderne Theateradaptionen auf den Leib geschrieben. Brasov: Universitatea Transilvania. 2016.

Scheller, Ingo: Szenisches Spiel. Handbuch für die pädagogische Praxis. Berlin: Cornelsen Verlag 1998.

Stănescu, Mirona: Theaterpädagogik in Rumänien. Hamburg: Verlag Dr. Kovač Gmbh, 2012.

\section{Internetquellen:}

Darstellendes Spiel. In: Wörterbuch der Theaterpädagogik. Hrsg. von Deutsches Archiv für Theaterpädagogik, DATP, Schibri Verlag 2003. Unter: http://www.archiv-datp.de/ worterbuch-darstellendes-spiel/ (Zugriff am 27.06.2019). Hagl, Elisabeth: Theaterpädagogik im Kontext einer lebendigen Lernkultur. Magisterarbeit im Fach Pädagogik an der Ludwig-Maximilian-Universität München, vorgelegt am 04.10.2006, unter: https://www.yumpu.com/de/document/ 
$\mathrm{read} / 3541843 /$ theaterpadagogik-im-kontext-einer-lebendigen-lernkultur (Zugriff am 21.06.2019).

Hoffmann, Klaus/ Radermacher, Norbert/ Reiss, Joachim u.a.: Bund Deutscher Amateurtheater: Resolution ,Kinder spielen Theater". In: Deutscher Kulturrat (Hrsg.): Kulturelle Bildung in der Bildungsreformdiskussion. 2005, S. 418419, unter: https://www.kulturrat.de/wp-content/uploads/ 2016/04/konzeption-kb3.pdf (Zugriff am 27.06.2019).

Puchianu, Carmen: Das darstellende Spiel und sein Einsatz im Germanistikunterricht am Beispiel eines Projektes mit Kronstädter Germanistikstudierenden. In: Temeswarer Beiträge zur Germanistik, Heft 6. Temeswar: Mirton Verlag 2008, S. 135-147, http://www.diacronia.ro/ro/indexing/de tails/A13124/pdf (Zugriff am 28.06.2019). 